\title{
Sublobar resection in high-risk patients for lobectomy: current and future strategy
}

\author{
Daniel P. Dolan, Scott J. Swanson \\ Division of Thoracic Surgery, Brigham and Women's Hospital, Boston, MA 02215, USA
}

Correspondence to: Dr. Daniel P. Dolan, Division of Thoracic Surgery, Brigham and Women's Hospital, 75 Francis St., Boston, MA 02215, USA. E-mail: ddolan7@bwh.harvard.edu

\begin{abstract}
How to cite this article: Dolan DP, Swanson SJ. Sublobar resection in high-risk patients for lobectomy: current and future strategy. Mini-invasive Surg 2021;5:3. http://dx.doi.org/10.20517/2574-1225.2020.101
\end{abstract}

Received: 27 Oct 2020 First Decision: 26 Nov 2020 Revised: 8 Dec 2020 Accepted: 14 Dec 2020 Published: 7 Jan 2021

Academic Editor: Noriyoshi Sawabata Copy Editor: Whitney Xu Production Editor: Jing Yu

\begin{abstract}
Surgical resection by lobectomy is the gold standard of therapy for early stage non-small cell lung cancer. However, not all patients are medically fit to undergo surgery. In patients considered high-risk for lobectomy, alternative strategies have been developed including radiofrequency ablation, cryoablation, microwave ablation, stereotactic radiation therapy, wedge resection, and segmentectomy. This work reviews the definition of high-risk, and the outcomes that have been associated with each treatment technique. Some technical points regarding wedge resection versus segmentectomy are noted. Future directions are discussed in the context of treatment for patients considered at high-risk for lobectomy.
\end{abstract}

Keywords: Non-small cell lung cancer, sublobar resection, surgical technique, high-risk patients

\section{INTRODUCTION}

This work is intended to review the current literature surrounding the definition of a patient with lungcancer who is considered high-risk for lobectomy, discuss different treatment modalities and their outcomes for these patients, and note some potential future directions and their benefits to high-risk patients. PubMed and EMBASE were reviewed and works were included based on relevance. Previous work in this field has involved clinical trials to determine patients who are considered high-risk, their results with sublobar resection, radiofrequency ablation (RFA), cryoablation, microwave ablation (MWA), stereotactic body radiation therapy (SBRT), and comparisons of these alterative techniques against sublobar resection.

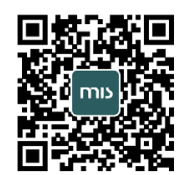


Technical aspects of wedge resection and segmentectomy are discussed for high-risk patients, and future directions of lung cancer treatment that could specifically benefit high-risk patients are noted.

\section{DEFINITION OF HIGH-RISK}

One of the most used definitions for high-risk patients come from the American College of Surgeons Oncology Group (ACOSOG) Z4032 trial of stage I non-small cell lung cancer (NSCLC), with tumors $\leq 3$ $\mathrm{cm}$, that focused on clinical details to define high risk $^{[1]}$. Patients were considered high-risk for sublobar resection, or sublobar resection with brachytherapy, if their pulmonary function tests (PFTs) showed a Forced Expiratory Volume in 1 second $(\mathrm{FEV} 1) \leq 50 \%$ of predicted, or if their Diffusing Capacity for Carbon Monoxide (DLCO) was $\leq 50 \%$ of predicted, or if they met two of the following criteria: age $\geq 75$ years, FEV1 51\%-60\% predicted, DLCO 51\%-60\%, diagnosed with pulmonary hypertension (pulmonary artery systolic greater $>40 \mathrm{mmHg}$ ) as estimated by echocardiography or right heart catheterization, left ventricular ejection fraction $\leq 40 \%$, resting or exercise arterial $\mathrm{pO}_{2} \leq 55 \mathrm{mmHg}$ or $\mathrm{SpO}_{2} \leq 88 \%, \mathrm{pCO}_{2}>$ $45 \mathrm{mmHg}$, or Modified Medical Research Council Dyspnea Scale score $\geq 3$.

However, while ACOSOG Z4032 provides a precise definition, controversy still exists. Puri et al. ${ }^{[2]}$ reported the non-propensity score matched findings of their review of 1066 patients from the Washington University School of Medicine. They found that perioperative outcomes for the high-risk group by ACOSOG Z4032 were not different from normal-risk patients - respiratory failure, $4 \%(7 / 194)$ in high risk vs. 5\% (41/872) in normal risk $(P=0.70)$; prolonged air leak of $>5$ days, $8 \%(16 / 194)$ in high risk $v s .6 \%(54 / 872)$ in normal risk $(P=0.36)$; and 30 day/hospital mortality $1 \%(2 / 194)$ in high risk $v s .2 \%(14 / 872)$ in normal risk $(P=$ 0.75). The most recent National Comprehensive Cancer Network NSCLC guidelines focus on a definition of high-risk that is aimed at risk of recurrence and leaves the definition of 'operative' high-risk unresolved ${ }^{[3]}$.

\section{PERI-OPERATIVE OUTCOMES OF HIGH-RISK PATIENTS UNDERGOING SUBLOBAR RESECTION}

Fernando et al. ${ }^{[4]}$ reported perioperative outcomes for their high-risk patients in 2011. Three deaths $(1.4 \%$, $3 / 222$ ), one in the sublobar resection group and two in the sublobar resection with brachytherapy group, occurred within 30 days. Three more deaths occurred by 90 days $(2.7 \% 6 / 222)$, and four of the deaths within 90 days were attributed to the surgery performed. Kent et al ${ }^{[5]}$ provided a further operative and pathologic analysis of this patient group in 2013. When segmentectomy ( $n=57$ patients) was compared to wedge resection ( $n=153$ patients), they found that segmentectomies had better margin size than wedge resections, median $1.5 \mathrm{~cm}$ (range $0.1-6.5 \mathrm{~cm})$ vs. $0.8 \mathrm{~cm}(0-3.6 \mathrm{~cm}), P=0.0001$; greater number of lymph node stations sampled, median $3(0-6) v s .1$ (0-6), $P<0.0001$; and greater number of lymph nodes removed, median 4 (0-20) vs. 1 (0-23), $P<0.0001$.

Sancheti et al. ${ }^{[6]}$ reported on their institution's experience with 'high-risk' patients defined by ACOSOG z4032. The study focused on Stage I NSCLC and, in their sub-analysis of patients who underwent sublobar resection, reported shorter operative time in the high-risk group $v s$. standard risk group, median 89.0 min (range 64.0-110.0) vs. 112.5 min (74.0-145.5), $P=0.04$; but longer length of stay, median 4 days (3-7) in the high risk group $v s$. median 3 days $(2-5)$ in the standard risk group, $P=0.003$. They found no statistical difference in total patient numbers with major morbidity, $12.3 \%$ (7/57) high risk group vs. $6.7 \%(4 / 40)$ standard risk group, $P=0.39$; but, noted more patients with minor morbidity in the high-risk group, $43.9 \%$ (25/57) vs. $20 \%(12 / 60)$ in the standard risk group, $P=0.02$. The 3 -year survival from sublobar resection was worse for high risk patients than standard risk patients, $57 \%$ s. $71 \%$, but not statistically significant, $P$ $=0.15$. 
Lastly, Puri et al.'s ${ }^{[2]}$ sub-analysis of their high-risk patients who underwent sublobar resection found no differences in perioperative outcomes be tween high-risk patients $(n=72)$ and normal-risk patients $(n=$ 112). Atrial fibrillation was slightly more common in the high-risk group than normal-risk, $11 \%(8 / 72) v s$. $6 \%$ (7/112), $P=0.28$, but this was potentially due to low event rates in both groups. They did not report the sublobar resection group survival Kaplan-Meier curves, but noted on logistic regression analysis that ACOSOG Z4032 high-risk status was not associated with the risk of perioperative complications (data not provided in their manuscript $)^{[1,2]}$.

\section{THERAPEUTIC CHOICE FOR OPTIMAL LONG-TERM OUTCOMES OF HIGH-RISK PATIENTS}

Since Ginsberg et al.'s ${ }^{[7]} 1995$ report on the Lung Cancer Study Group's randomized control trial of lobectomy vs. limited resection for T1 NSCLC, lobectomy has remained the gold standard for resection of early stage lung cancer. However, the current NCCN guidelines state that anatomic pulmonary resection is the preferred method for the majority of patients with NSCLC ${ }^{[3,7]}$. The NCCN guidelines further elaborate on sublobar resection as being appropriate in the setting of "poor pulmonary reserve or other major comorbidity that contraindicates lobectomy", while noting that SBRT is recommended for medically inoperable patients or patients who refuse surgery ${ }^{[3]}$. As there is no clear definition for "high-risk" patients, the choice of therapy for high-risk patients remains the purview of the clinicians treating the patient. Ablation techniques, SBRT, and lobectomy continue to be options for high-risk patients, in addition to sublobar resection.

Multiple ablation techniques have been reported for lung cancer including RFA, microwave ablation, and cryoablation $^{[8-10]}$. In 2005, Fernando et al ${ }^{[8]}$ reported RFA as an alternative for patients with peripheral lung cancer who are not surgical candidates. In their initial 18 patient series with 21 total tumors, they treated patients of all stages with a median tumor size of $1.8 \mathrm{~cm}$ (range $1.2-4.5 \mathrm{~cm}$ ). In this broad patient set, they noted a mean progression-free survival of 16.8 months. In 2007, Simon et al. ${ }^{[11]}$ published their 153 patient series from 1998-2005 that reviewed the outcomes of patients treated with RFA who were refused surgery or were not deemed suitable as surgical candidates. The 5-year survival rate for stage I NSCLC was $27 \%$, with local progression-free rate for tumors $\leq 3 \mathrm{~cm}$ equal to $47 \%$, and for $>3 \mathrm{~cm}$ equal to $25 \%$. A recent review of the National Cancer Database compared SBRT to RFA for stage I NSCLC $(4,454$ SBRT vs. 335 RFA patients ${ }^{[12]}$. RFA patients were noted to have more comorbidities than SBRT patients. They performed a propensity score matching and found no difference in the overall survival rate (OS) at 1-, 3-, and 5- years (31.9\% SBRT vs. 27.1\% RFA, $P=0.835)$.

MWA is another thermal ablation technique that uses high temperature to destroy tumors ${ }^{[9]}$. Zhong et al..$^{[9]}$ reported on 113 patients who underwent microwave ablation; 35 patients had early stage disease and 78 patients had late stage lung cancer. 10.6\% (12/133) of all patients had a pneumothorax after the procedure, but no intraoperative or perioperative deaths were observed. At 3 years, they reported that the survival of the early stage group was $84.7 \%$, in comparison to $71.7 \%$ in the advanced stage group, $P=0.576$. Zhao et al ${ }^{[13]}$ reported a longer-term follow-up (out to 5 years) of 34 early stage patients (T1a-T3NoMo). Pneumothorax was noted in 24 cases (59\%) with 6 cases requiring chest tube insertion (15\%). Their 5-year overall survival rate was $46.7 \%$. Yuan et al. ${ }^{[14]}$ performed a meta-analysis of 53 studies to compare outcomes of RFA with MWA for primary lung cancer and pulmonary metastases. They found a pooled pneumothorax rate of $34.3 \%$ (95\%CI: $25.9 \%-43.1 \%$ ) in the RFA group vs. $33.9 \%$ (95\%CI: $23.8 \%-44.8 \%$ ) in the MWA group, $P=0.957$. Severe pneumothorax that required intervention occurred in $12.3 \%$ of patients (95\%CI: 6.8\%-19.1\%) in the RFA group and in $11.0 \%$ of patients (95\%CI: $4.5 \%-19.7 \%$ ) in the MWA group, $P$ $=0.797$. Based on the 8 studies for RFA and 6 studies for MWA, they found comparable median OS for the 2 groups, RFA 28.4 months (95\%CI: 20.9-35.8) vs. MWA 24.4 months (95\%CI: 16.9-31.8). 
Cryoablation is the other common ablative technique and works by creating a freezing zone that first freezes the extracellular fluid and then the intracellular fluid, causing cellular and tissue destruction during multiple cycles with temperature ranges typically in the $-20 \mathrm{C}$ to $-40 \mathrm{C}$ range $\mathrm{e}^{[15,16]}$. For Stage I NSCLC, Yamauchi et al ${ }^{[17]}$ reported a 3-year overall survival of $88 \%$ and a disease-free 3-year survival of $67 \%$, while Moore et al. ${ }^{[18]}$ reported a 5-year overall survival of $67.8 \% \pm 15.3$, cancer-specific survival of $56.6 \%$ \pm 16.5 , and 5 -year progression-free survival rate of $87.9 \% \pm 9$. Yamauchi reported pneumothorax in $28 \%$ cases (7/25) vs. Moore's report of 51.0\% (24/45); and each reported 1 case requiring chest tube insertion. Zemlyak et al. ${ }^{[19]}$ performed a small retrospective, non-propensity matched comparison between sublobar resection $(n=25)$, radiofrequency ablation $(n=12)$ and percutaneous cryoablation $(n=27)$ and found that the 3 -year overall survival was $87.1 \%, 87.5 \%$, and $77 \%$, respectively, $P>0.05$. Additionally, the 3 -year cancer-specific and cancer-free survival for sublobar resection, radiofrequency ablation, and percutaneous cryoablation groups was $90.6 \%$ and $60.8 \%, 87.5 \%$ and $50 \%$, and $90.2 \%$ and $45.6 \%$, respectively with $P>0.05$ for intergroup comparisons of 3-year cancer specific survival and 3-year cancer free survival. They noted that the lack of significance was likely due to a small sample size.

The American Society of Radiation Oncology defines SBRT as ablation radiation doses in 1-5 fractions with high conformal techniques ${ }^{[20]}$. They note in these consensus guidelines that stage I NSCLC patients with "high operative risk" should be offered SBRT as an alternative to sublobar resection, but the longer-term outcomes over 3 years are not well-established. Some of the longest survival data for SBRT comes from a follow-up of the North Central Cancer Trials Group N0927 randomized phase II study, comparing 34 Gy vs. 48 Gy SBRT for medically inoperable stage I peripheral NSCLC ${ }^{[21-22]}$. They found that the 5-year overall survival in the 34 Gy and 48 Gy groups were $29.6 \%$ (95\%CI: $16.2 \%-44.4 \%$ ) and $41.1 \%$ (95\%CI: $26.6 \%$ $55.1 \%$ ) respectively. Progression-free survival at 5 years was $19.1 \%$ (95\%CI: 8.5\%-33.0\%) and $33.3 \%$ (95\%CI: $20.2 \%-47.0 \%$ ) for the 34 Gy and 48 Gy groups respectively. A recent systematic review and pooled analysis compared RFA to SBRT, and found that SBRT has better 5 year local tumor control rate, $42 \%$ (95\%CI: $30 \%$ 54\%) RFA vs. $86 \%$ (95\%CI: $85 \%-88 \%$ ) SBRT P< 0.001 ; but similar OS, $32 \%$ (95\%CI: $22 \%-43 \%$ ) for RFA vs. $40 \%$ (95\%CI: $36 \%-45 \%$ ) for SBRT $P=0.41^{[23]}$. In 2019, Ager et al ${ }^{[24]}$ reviewed the National Cancer Database and compared 14,651 SBRT patients to 1141 patients who underwent some form of percutaneous local tumor ablation therapy (LTA). After propensity score matching, their Cox modeling found a hazard ratio of 0.83, 95\%CI: 0.73-0.94, $P=0.002$, showing improved survival for SBRT patients. Adjusted rates of OS at 5 years were $31.0 \%$ and $26.2 \%$ for SBRT and LTA, respectively. Chi et al. ${ }^{[25]}$ also reviewed the National Cancer Database and compared SBRT to multiple different forms of surgery for early stage lung cancer. They found that the 5-year overall survival for the resection groups ranged from $48.1 \%$ (wedge resection) to $64.6 \%$ (lobectomy), compared to $30.4 \%$ in the SBRT cohort, $P<0.01$ for each resection type $v s$. SBRT. Their Cox model hazard ratios for wedge resection, segmentectomy, and lobectomy compared to SBRT demonstrated improved overall survival with surgery with values from 0.55 (wedge resection) to 0.40 (lobectomy), each $P$ value $<0.01$.

In terms of surgical treatment, Jensik et al. ${ }^{[26]}$ were the first to propose segmentectomy as an appropriate alternative to lobectomy for small-sized lung cancers. Since then, the debate has continued with findings for and against this in randomized trials, large database studies, and meta-analysis reviews, with lobectomy continuing as the standard of care with allowances for sublobar resection of high-risk $\operatorname{cases}^{[3,7,27,28]}$. Relatively few studies have focused on direct comparisons of surgical options in high-risk patients. Ijsseldijk et al. ${ }^{[28]}$ recently published a comprehensive systematic review and meta-analysis of 100 studies comparing SBRT, sublobar resection, and lobectomy. In this work, they found that lobar resection had a 5 -year OS of $74 \%$ $[0.69,0.78]$, sublobar resection had a 70\% OS [0.64, 0.77], and SBRT had a 46\% OS [0.35, 0.57], with both surgical survivals statistically better compared to SBRT, both $P<0.01$. Disease-free survival at 5 years in patients who had lobar resection was $76 \%[0.71,0.82]$, sublobar resection was $71 \%[0.67,0.76]$, and SBRT was $46 \%[0.35,0.57]$, with both surgical survivals statistically better compared to SBRT, $P<0.01$. However, 
this work was not specified to include only high-risk patients. Hou et al. ${ }^{[29]}$ performed a specified metaanalysis directly comparing segmentectomy to sublobar resection for high-risk patients in 9 studies. They noted heterogeneous definitions for what qualified as high-risk, but most studies had followed Fernando et al.' ${ }^{[1]}$ criteria from their 2011 work mentioned earlier in this review. For OS, Hou et al. ${ }^{[29]}$ included 7 studies, and on meta-analysis found that the hazard ratio for segmentectomy compared to wedge resection for stage I NSCLC was 0.80 in favor of improved OS with segmentectomy compared to wedge resection [95\%CI: 0.68-0.93; $P=0.004]$. On subgroup analysis, there was comparable OS for stage I tumors $\leq 2 \mathrm{~cm}$; however, the hazard ratio favored improved OS with segmentectomy compared to wedge resection, 0.39 [95\%CI: 0.15-1.02; $P=0.06]^{[29]}$. Cancer-specific survival also favored segmentectomy over wedge resection, hazard ratio 0.42 [95\%CI: $0.20-0.88 ; P=0.02$ ]. They were unable to fully assess disease-free survival as only 3 studies reported data that was usable for comparison.

Unfortunately, even with all this data, the best option for high-risk patients who can undergo limited resection, but not full lobectomy, remains unclear. Three randomized control trials are ongoing comparing lobectomy to sublobar resection for early stage NSCLC, $\leq 2 \mathrm{~cm}$ with No lymph node status ${ }^{[30-32]}$. All three studies have reported their peri-operative safety results and found no substantial differences. Suzuki et al. ${ }^{[30]}$ noted a higher airleak rate in their 552 segmentectomy patients compared to their 554 lobectomy patients, $6.5 \%$ vs. 3.8\%, $P=0.04$. However, Altorki et al. $\mathrm{s}^{[31]}$ report did not note an increased airleak in their sublobar resection group, 340 patients total, compared to their 357 lobectomy patients. This was despite including wedge resections and segmentectomies in their sublobar resection group; 201 wedge resection patients and 139 lobectomy patients ${ }^{[31]}$. Stamatis et al ${ }^{[32]}$ noted equal rates of prolonged air leak in their 53 segmentectomy patients compared to their 54 lobectomy patients. Until the long-term outcomes of these randomized control trials are evaluated, the choice of therapy should be determined by a multidisciplinary team. Surgical resection, when feasible and preferably segmentectomy, remains the recommended treatment if lobectomy is not possible ${ }^{[3]}$.

\section{SUBLOBAR RESECTION TECHNICAL POINTS: WEDGE RESECTION VS SEGMENTECTOMY}

Patient selection remains of paramount importance for surgical procedure choice. Wedge resection and segmentectomy are most appropriate for smaller, peripherally located lesions away from the hilum of the lung. Segmentectomy should be favored when possible given its respect for anatomic planes, but comes with a caveat. The target lesion mustlie within the boundaries of one segment or group of segments. One of the authors has written extensively regarding this process and reported that patients with lesions under $2 \mathrm{~cm}$ that are resected with segmentectomy have no difference in outcomes compared to the patients treated with lobectomy ${ }^{[33-35]}$. Segmentectomies are more technically challenging as the surgeon must create a fissure between segments and then dissect out and ligate the segmental vessels and bronchus. Wedge resection is performed without respect to anatomic planes or specific vessels, but can be useful when the target lesion is very small ( $1 \mathrm{~cm}$ or less), subpleural, or crosses segmental borders. Care should be taken to ensure that the margins from the edge of the tumor to the final staple line are appropriately wide to minimize recurrence and that adequate lymph nodes are removed to ensure accurate staging ${ }^{[36-38]}$.

\section{FUTURE DIRECTIONS}

Radiomics is a rapidly growing field in which radiographic images are used to determine features such as lesion shape, volume, texture, attenuation, and other factors that are not readily apparent or are too difficult for an individual radiologist to assess visually or qualitatively ${ }^{[39]}$. Radiomics is being studied to predict histologic subtypes, specific mutations, and benefit of adjuvant chemotherapy after resection ${ }^{[40-42]}$. Radiomics has already been used to predict OS in NSCLC; specifically, the recurrence of NSCLC after $\mathrm{SBRT}^{[43-45]}$. Radiomics may even be able to predict survival based on resection type and offer high-risk patients more tailored care. 
Another advancement lies in improved lesion targeting for NSCLC that is not located at the outermost periphery. Image-guided video-assisted thoracoscopic surgery (iVATS) is an emerging technology that is being developed to allow localization of non-palpable lesions ${ }^{[4]}$. Early reports indicate excellent rates of localization using modifications of previously developed techniques with wires, microcoils, and indocyanine green with near-infrared imaging ${ }^{[46-48]}$. These lesions are then removed with sublobar resections. Of note, the use of this technology requires a hybrid operating room equipped with a CT scanner, which is not available at all facilities. By being able to remove deeper lesions while still performing wedge resection or segmentectomy, iVATS offers high-risk patients another surgical option for lesions that previously may have required lobectomy.

\section{CONCLUSION}

In summary, high-risk patients remain a poorly defined group, with patients typically defined as those with some degree of poor pulmonary function and often other significant functional or medical limitations. In these patients, surgical resection is the gold standard relative to SBRT and ablative techniques. Segmentectomy should be performed rather than wedge resection when feasible, and when lobectomy is not an option. Future developments in radiomics and iVATS technique may help further refine the optimal treatment approaches for high-risk patients.

\section{DECLARATIONS}

\section{Authors' contributions}

Made substantial contributions to the conception and design of the study, performed data analysis and interpretation, data acquisition, as well as provided administrative, technical and material support: Dolan D, Swanson S

\section{Availability of data and materials}

Not applicable.

\section{Financial support and sponsorship}

None.

\section{Conflicts of interest}

Dr. Swanson reports receipt of honoraria from Covidien and Ethicon for speaking and consulting services.

\section{Ethical approval and consent to participate}

Not applicable.

\section{Consent for publication}

Not applicable.

\section{Copyright}

(c) The Author(s) 2021.

\section{REFERENCES}

1. Fernando HC, Landreneau RJ, Mandrekar SJ, et al. The impact of adjuvant brachytherapy with sublobar resection on pulmonary function and dyspnea in high-risk patients with operable disease: preliminary results from the American College of Surgeons Oncology Group Z4032 trial. J Thorac Cardiovasc Surg 2011;142:554-62.

2. Puri V, Crabtree TD, Bell JM, et al. National cooperative group trials of "high-risk" patients with lung cancer: are they truly "high-risk"? Ann Thorac Surg 2014;97:1678-83.

3. National Comprehensive Cancer Network. Non-Small Cell Lung Cancer (Version 7.2020) Available from: https://www.ncen.org/ 
professionals/physician_gls/pdf/nscl.pdf [Last accessed on 18 Dec 2020.]

4. Fernando HC, Landreneau RJ, Mandrekar SJ, et al. Thirty- and ninety-day outcomes after sublobar resection with and without brachytherapy for non-small cell lung cancer: results from a multicenter phase III study. J Thorac Cardiovasc Surg 2011;142:1143-51.

5. Kent M, Landreneau R, Mandrekar S, et al. Segmentectomy versus wedge resection for non-small cell lung cancer in high-risk operable patients. Ann Thorac Surg 2013;96:1747-54.

6. Sancheti MS, Melvan JN, Medbery RL, et al. Outcomes after surgery in high-risk patients with early stage lung cancer. Ann Thorac Surg 2016;101:1043-50.

7. Ginsberg RJ, Rubinstein LV. Randomized trial of lobectomy versus limited resection for T1 N0 non-small cell lung cancer. Ann Thorac Surg 1995;60:615-22.

8. Fernando HC, De Hoyos A, Landreneau RJ, et al. Radiofrequency ablation for the treatment of non-small cell lung cancer in marginal surgical candidates. J Thorac Cardiovasc Surg 2005;129:639-44.

9. Zhong L, Sun S, Shi J, et al. Clinical analysis on 113 patients with lung cancer treated by percutaneous CT-guided microwave ablation. $J$ Thorac Dis 2017;9:590-7.

10. Wang H, Littrup PJ, Duan Y, Zhang Y, Feng H, Nie Z. Thoracic masses treated with percutaneous cryotherapy: initial experience with more than 200 procedures. Radiology 2005;235:289-98.

11. Simon CJ, Dupuy DE, DiPetrillo TA, et al. Pulmonary radiofrequency ablation: long-term safety and efficacy in 153 patients. Radiology 2007;243:268-75.

12. Lam A, Yoshida EJ, Bui K, Fernando D, Nelson K, Abi-Jaoudeh N. A national cancer database analysis of radiofrequency ablation versus stereotactic body radiotherapy in early-stage non-small cell lung cancer. J Vasc Interv Radiol 2018;29:1211-7.

13. Zhao H, Steinke K. Long-term outcome following microwave ablation of early-stage non-small cell lung cancer. J Med Imaging Radiat Oncol 2020;64:787-93.

14. Yuan Z, Wang Y, Zhang J, Zheng J, Li W. A meta-analysis of clinical outcomes after radiofrequency ablation and microwave ablation for lung cancer and pulmonary metastases. J Am Coll Radiol 2019;16:302-14.

15. Inoue M, Nakatsuka S, Jinzaki M. Cryoablation of early-stage primary lung cancer. Biomed Res Int 2014;2014:521691.

16. Zhang YS, Niu LZ, Zhan K, et al. Percutaneous imaging-guided cryoablation for lung cancer. J Thorac Dis 2016;8:S705-9.

17. Yamauchi Y, Izumi Y, Hashimoto K, et al. Percutaneous cryoablation for the treatment of medically inoperable stage I non-small cell lung cancer. PLoS One 2012;7:e33223.

18. Moore W, Talati R, Bhattacharji P, Bilfinger T. Five-year survival after cryoablation of stage I non-small cell lung cancer in medically inoperable patients. J Vasc Interv Radiol 2015;26:312-9.

19. Zemlyak A, Moore WH, Bilfinger TV. Comparison of survival after sublobar resections and ablative therapies for stage I non-small cell lung cancer. J Am Coll Surg 2010;211:68-72.

20. Videtic GMM, Donington J, Giuliani M, et al. Stereotactic body radiation therapy for early-stage non-small cell lung cancer: executive summary of an ASTRO evidence-based guideline. Pract Radiat Oncol 2017;7:295-301.

21. Videtic GM, Hu C, Singh AK, et al. A randomized phase 2 study comparing 2 stereotactic body radiation therapy schedules for medically inoperable patients with stage i peripheral non-small cell lung cancer: NRG oncology RTOG 0915 (NCCTG N0927). Int J Radiat Oncol Biol Phys 2015;93:757-64.

22. Videtic GM, Paulus R, Singh AK, et al. Long-term follow-up on NRG oncology RTOG 0915 (NCCTG N0927): a randomized phase 2 study comparing 2 stereotactic body radiation therapy schedules for medically inoperable patients with stage I peripheral non-small cell lung cancer. Int J Radiat Oncol Biol Phys 2019;103:1077-84.

23. Bi N, Shedden K, Zheng X, Kong FS. Comparison of the effectiveness of radiofrequency ablation with stereotactic body radiation therapy in inoperable stage i non-small cell lung cancer: a systemic review and pooled analysis. Int J Radiat Oncol Biol Phys 2016;95:1378-90.

24. Ager BJ, Wells SM, Gruhl JD, et al. Stereotactic body radiotherapy versus percutaneous local tumor ablation for early-stage non-small cell lung cancer. Lung Cancer 2019;138:6-12.

25. Chi A, Fang W, Sun Y, Wen S. Comparison of long-term survival of patients with early-stage non-small cell lung cancer after surgery vs stereotactic body radiotherapy. JAMA Netw Open 2019;2:e1915724.

26. Jensik RJ, Faber LP, Milloy FJ, Monson DO. Segmental resection for lung cancer. A fifteen-year experience. J Thorac Cardiovasc Surg 1973;66:563-72.

27. Altorki NK, Yip R, Hanaoka T, et al; I-ELCAP investigators. Sublobar resection is equivalent to lobectomy for clinical stage 1A lung cancer in solid nodules. J Thorac Cardiovasc Surg 2014;147:754-62.

28. Ijsseldijk MA, Shoni M, Siegert C, et al. Oncologic outcomes of surgery versus sbrt for non-small-cell lung carcinoma: a systematic review and meta-analysis. Clin Lung Cancer 2020:S1525-7304(20)30140-6.

29. Hou B, Deng XF, Zhou D, Liu QX, Dai JG. Segmentectomy versus wedge resection for the treatment of high-risk operable patients with stage I non-small cell lung cancer: a meta-analysis. Ther Adv Respir Dis 2016;10:435-43.

30. Suzuki K, Saji H, Aokage K, et al. Comparison of pulmonary segmentectomy and lobectomy: safety results of a randomized trial. $J$ Thorac Cardiovasc Surg 2019;158:895-907.

31. Altorki NK, Wang X, Wigle D, et al. Perioperative mortality and morbidity after sublobar versus lobar resection for early-stage nonsmall-cell lung cancer: post-hoc analysis of an international, randomised, phase 3 trial (CALGB/Alliance 140503). Lancet Respir Med 2018;6:915-24.

32. Stamatis G, Leschber G, Schwarz B, et al. Perioperative course and quality of life in a prospective randomized multicenter phase III trial, comparing standard lobectomy versus anatomical segmentectomy in patients with non-small cell lung cancer up to $2 \mathrm{~cm}$, stage IA (7th 
edition of TNM staging system). Lung Cancer 2019;138:19-26.

33. Shapiro M, Weiser TS, Wisnivesky JP, Chin C, Arustamyan M, Swanson SJ. Thoracoscopic segmentectomy compares favorably with thoracoscopic lobectomy for patients with small stage I lung cancer. J Thorac Cardiovasc Surg 2009;137:1388-93.

34. Bilgi Z, Swanson SJ. Current indications and outcomes for thoracoscopic segmentectomy for early stage lung cancer. $J$ Thorac Dis 2019;11:S1662-9.

35. McKenna RJ, Mahtabifard A, Swanson SJ. Atlas of minimally invasive thoracic surgery (VATS). 1st ed. Philadelphia: Elsevier. 2011.

36. Mohiuddin K, Haneuse S, Sofer T, et al. Relationship between margin distance and local recurrence among patients undergoing wedge resection for small ( $\leq 2 \mathrm{~cm}$ ) non-small cell lung cancer. J Thorac Cardiovasc Surg 2014;147:1169-75.

37. Khullar OV, Liu Y, Gillespie T, et al. Survival after sublobar resection versus lobectomy for clinical stage ia lung cancer: an analysis from the national cancer data base. $J$ Thorac Oncol 2015;10:1625-33.

38. Wolf AS, Swanson SJ, Yip R, et al; I-ELCAP investigators. The impact of margins on outcomes after wedge resection for stage i nonsmall cell lung cancer. Ann Thorac Surg 2017;104:1171-8.

39. Hassani C, Varghese BA, Nieva J, Duddalwar V. Radiomics in pulmonary lesion imaging. AJR Am J Roentgenol 2019;212:497-504.

40. Wu W, Parmar C, Grossmann P, et al. Exploratory Study to identify radiomics classifiers for lung cancer histology. Front Oncol 2016;6:71.

41. Liu Y, Kim J, Balagurunathan Y, et al. Radiomic features are associated with egfr mutation status in lung adenocarcinomas. Clin Lung Cancer 2016;17:441-8.

42. Vaidya P, Bera K, Gupta A, et al. CT derived radiomic score for predicting the added benefit of adjuvant chemotherapy following surgery in stage I, II resectable non-small cell lung cancer: a retrospective multicohort study for outcome prediction. The Lancet Digital Health 2020;2:e116-28.

43. Gill RR, Barlow J, Jaklitsch MT, Schmidlin EJ, Hartigan PM, Bueno R. Image-guided video-assisted thoracoscopic resection (iVATS): translation to clinical practice-real-world experience. J Surg Oncol. 2020;121:1225-32.

44. Huynh E, Coroller TP, Narayan V, et al. Associations of radiomic data extracted from static and respiratory-gated CT scans with disease recurrence in lung cancer patients treated with SBRT. PLoS One 2017;12:e169172.

45. Mattonen SA, Palma DA, Johnson C, et al. Detection of local cancer recurrence after stereotactic ablative radiation therapy for lung cancer: physician performance versus radiomic assessment. Int J Radiat Oncol Biol Phys 2016;94:1121-8.

46. Gill RR, Barlow J, Jaklitsch MT, Schmidlin EJ, Hartigan PM, Bueno R. Image-guided video-assisted thoracoscopic resection (iVATS): translation to clinical practice-real-world experience. J Surg Oncol 2020;121:1225-32.

47. Chao YK, Leow OQY, Wen CT, Fang HY. Image-guided thoracoscopic lung resection using a dual-marker localization technique in a hybrid operating room. Surg Endosc 2019;33:3858-63.

48. Wen CT, Liu YY, Fang HY, Hsieh MJ, Chao YK. Image-guided video-assisted thoracoscopic small lung tumor resection using nearinfrared marking. Surg Endosc 2018;32:4673-80. 